\title{
Systemic inflammatory disorders in patients admitted for aseptic meningitis
}

\author{
Authors: Marine Boudot de la Motte, ${ }^{\mathrm{A}}$ Rachid Abbas, ${ }^{\mathrm{B}}$ Fanny Jouan, ${ }^{\mathrm{C}}$ Damien van Gysel, ${ }^{\mathrm{D}}$ \\ Marie Paule Chauveheid, ${ }^{\mathrm{E}}$ Thomas Papo ${ }^{\mathrm{F}}$ and Karim Sacre ${ }^{\mathrm{G}}$
}

Acute meningitis can be the first manifestation of an underlying systemic inflammatory disorder (SID). In the current study, we aimed to identify clinical indicators for SIDs in patients admitted for acute aseptic meningitis. All patients hospitalised for acute aseptic meningitis over a 4-year period in a department of internal medicine were included retrospectively. Patients with neoplastic meningitis were excluded. Extraneurological signs were recorded using a systematic panel. Systemic inflammatory disorder diagnosis was made according to current international criteria. Forty-three (average age 46 years [range 19-82 years], 60\% females) consecutive patients were analysed retrospectively. Of these, 23 patients had an SID (mostly sarcoidosis and Behçet's disease). Multiple logistic regression analysis showed that the probability of an SID was $93.7 \%$ in patients with both neurological and extraneurological signs, but $14.9 \%$ in patients with neither neurological nor extraneurological signs. In conclusion, clinical sorting according to both neurological and extraneurological signs could help to identify patients with acute aseptic meningitis caused by an SID.

KEYWORDS: Aseptic meningitis, systemic inflammatory disorders, diagnosis, hospitalised patients, internal medicine

\section{Introduction}

The most frequent causes of aseptic meningitis are systemic inflammatory disorders (SIDs), drugs and cancer. ${ }^{1,2}$ In SIDs, central nervous system (CNS) involvement, including aseptic meningitis, can herald the disease and stands as a major cause of morbidity and mortality. However, most studies consider only specific CNS manifestations in the context of separate entities, such as systemic lupus erythematosus (SLE), Sjögren's syndrome, Behçet's disease or sarcoidosis. ${ }^{3-10}$ To the best of our knowledge, no global

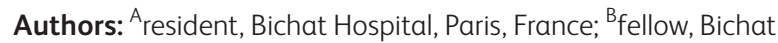
Hospital, Paris, France; C fellow, Bichat Hospital, Paris, France; Dhospital practitioner, Bichat Hospital, Paris, France; Ehospital practitioner, Bichat Hospital, Paris, France; F professor of medicine, Bichat Hospital, Paris, France; ${ }^{G}$ professor of medicine, Bichat Hospital, Paris, France approach has been designed to detect SIDs in patients with acute aseptic meningitis. By contrast, aseptic meningitis caused by SID is expected to be rare ${ }^{11}$ and a systematic extended etiological workup might seem unreasonable.

Our structured retrospective cohort study aimed to identify simple clinical indicators for SIDs in patients admitted for acute meningitis, once infection had been ruled out.

\section{Materials and methods}

\section{Patients}

All consecutive adult patients hospitalised from January 2010 to October 2014 for acute aseptic meningitis in a department of internal medicine (Bichat Hospital, Paris, France) were included. Aseptic meningitis was defined by the acute onset of meningeal irritation symptoms (headache and neck stiffness with or without fever), and a white cell count in the cerebrospinal fluid (CSF) greater than $5.0 / \mathrm{mm}^{3}$ without identification of bacterial, fungus or viral agents. ${ }^{12}$ Screening for pathogens included at least direct CSF Gram stain and culture, and polymerase chain reaction (PCR) assays for the detection of herpes simplex virus (HSV) 1 and 2 and enterovirus. Additional tests (PCR for varicella zoster virus, cytomegalovirus, JC virus, Epstein-Barr virus, human herpesvirus 6, Toxoplasma gondii, Tropheryma whipplei and Mycobacterium tuberculosis, and Indian ink test for Cryptococcus) were performed in some cases based on clinical findings. Exclusion criteria included an inability to confirm meningitis (ie inability to confirm CSF pleocytosis after consulting medical records and microbiological database), or a final diagnosis of neoplastic meningitis.

\section{Data collection}

International Classification of Disease codes (ICD-10) for aseptic meningitis, including A86, A170, A872/9, A858, G009, G020, G030/1/2/8/9, G040/8/9, G051/2/8, G92, G379 and G934, were used to screen patients. Data were extracted from the French Diagnosis Related Groups (DRG)-based information system (PMSI) databases. Information regarding eligibility conditions and exclusion criteria were obtained from medical records. The clinical neurological status of each patient was obtained from medical records, and divided into in six parameters: cognitive disorders, seizure, focal neurological signs, coma, abnormal involuntary movement and delirium. Cell counts, protein and glucose levels in the CSF were obtained from the biological database. High 
Box 1. Extraneurological signs systematically

assessed by a standardised medical history taking in

all patients admitted with acute aseptic meningitis

$>$ Alopecia

$>$ Aphthous ulcers

Arthralgia

$>$ Erythema nodosum

Folliculitis

$>$ Malar rash

$>$ Myalgia

> Pain and redness of the eye with or without blurred vision

> Parotid gland swelling

Photosensitivity

Raynaud phenomenon

$>$ Sicca syndrome

Uveitis

protein levels in the CSF were defined as $>0.4 \mathrm{~g} / \mathrm{L}$, and low glucose levels as a reduction of $>50 \%$ compared with the serum glucose level. All brain and spine magnetic resonance images (MRIs) were reviewed. Abnormal findings were classified as: T2-weighted high signal lesions, pseudotumoural lesions, arachnoiditis, dilatation of ventricles and myelitis. Data on severity (ie transfer in the intensive care unit), treatment and follow-up were retrieved from medical records.

\section{Search for systemic inflammatory disorders}

Past medical history, background and extraneurological signs were analysed using a systematic panel (Box 1). A complete physical examination was performed in all cases. Standard blood tests included serum C-reactive protein (CRP) in all patients. More specific work-ups, such as serum auto-antibodies or fundoscopy, were performed as required based on the first set of results. A diagnosis was then confirmed or ruled out according to current international criteria.

\section{Ethical statement}

Our study was a retrospective human non-interventional study. According to the Public Health French Law (art L 1121-1-1, art L 1121-1-2), approval from the institutional review board and written consent are not required for human non-interventional studies. However, for ethical reasons, patients were informed that the data that were collected in their medical records might be used for research studies in accordance with privacy rules. The study protocol conformed to the ethical guidelines of the 1975 Declaration of Helsinki.

\section{Statistical analysis}

Data are expressed as mean plus standard deviation (SD) or frequency (percentage) as appropriate. For descriptive and analytical purposes, the study population was split into two groups: a first group of patients in whom an inflammatory or autoimmune disease was identified as the cause of meningitis (SID group) and a second group of patients in whom no such disease was identified (idiopathic group). The Student's T-test was used to compare age in both groups. The Fisher's exact test was used to compare dichotomous variables. Univariate logistic regression analyses were performed to evaluate the association between patient characteristics (age, sex, fever, neurological signs, extraneurological signs, CRP level in serum, and CSF analysis) and the etiology of meningitis (SIDs or idiopathic). A variance inflation factor was used to eliminate the least significant variables at risk for multicollinearity. Subsequently, a multiple logistic regression analysis using the factors selected in the univariate analysis with a 0.05 significance threshold was carried out to identify factors independently associated with the etiology of meningitis. A logistic regression model was used to compute the predicted probabilities of autoimmune etiology for meningitis of the study patients. A sensibility analysis was conducted using the lasso shrinkage method during the explanatory variable selection on our data set to assess the robustness of the model. Statistical analyses were performed with GraphPad Prism 5.01 software and R statistical software version 3.3 (R Foundation for statistical Computing, Vienna, Austria). P-values $<0.05$ were considered statistically significant.

\section{Results}

\section{Demographic characteristics}

From January 2010 to December 2014, 88 patients hospitalised in a department of internal medicine (Bichat Hospital, Paris, France) were eligible for the study according to the International Classification of Disease codes (ICD-10) for aseptic meningitis. Forty-five patients were excluded because of their final diagnosis, infection $(n=5)$ or neoplastic meningitis $(n=6)$, or when meningitis itself could not be confirmed after individual chart review $(n=34)$. Eventually, 43 patients were included (Fig 1).

Treatment at admission was cautiously analysed. No patient was treated with drugs known to induce aseptic meningitis, such as non-steroidal anti-inflammatory drugs, antibiotics or intravenous immunoglobulins. Twenty-six (60\%) patients were women. The mean age was 46 (19-82) years. Fifteen (34.9\%) patients had fever at diagnosis. Neurological signs suggesting meningoencephalitis were observed in most cases ( $n=27,62.7 \%$ ). Brain MRI was performed in 41 patients and was considered normal in only $11(26.8 \%)$ cases. Thirteen $(30.2 \%)$ patients were transferred to the intensive care unit. Chronic or recurrent meningitis occurred in 15 cases (34.9\%). Further details are provided in Tables 1 and 2.

\section{Systemic inflammatory disorders as a cause of acute aseptic meningitis}

Twenty-three (53.5\%) patients hospitalised for aseptic meningitis had an SID. Meningitis revealed the disease in 16 patients $(69.5 \%)$, including sarcoidosis ( $n=5)$, Behçet's syndrome $(n=3)$, Susac's syndrome $(n=3)$, SLE $(n=2)$, CNS primary angiitis $(n=2)$ and anti- $\mathrm{N}$-methyl-D-aspartate (NMDA) receptor encephalitis $(n=1)$. Seven patients had a systemic disorder that was already known at the time of meningitis diagnosis, including sarcoidosis 


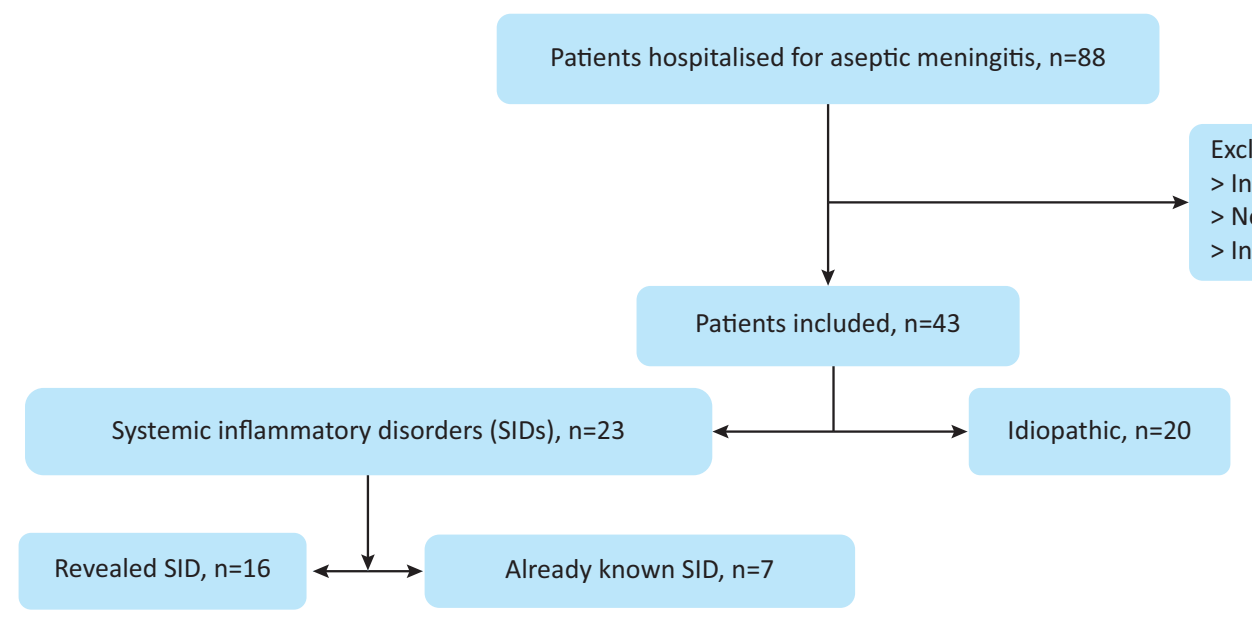

Fig 1. Flow chart of study patients. The inability to confirm meningitis refers to cases where cerebrospinal fluid (CSF) pleocytosis could not be confirmed after analysing medical records and a microbiological database. SIDs = systemic inflammatory disorders

Table 1. Patient characteristics at diagnosis

\begin{tabular}{|c|c|c|c|c|}
\hline & All $(n=43)$ & Idiopathic $(n=20)$ & $\operatorname{SID}(n=23)$ & $\mathrm{p}$-value \\
\hline Age, median (range) & $46(19-82)$ & $47.5(19-82)$ & $44(23-69)$ & 0.728 \\
\hline Female, n (\%) & $26(60)$ & $10(50)$ & $16(69.6)$ & 0.191 \\
\hline Fever, n (\%) & $15(34.9)$ & $9(45)$ & $6(26.1)$ & 0.194 \\
\hline Neurological signs, n (\%) & $27(62.7)$ & $9(45)$ & $18(78.3)$ & 0.024 \\
\hline Cognitive disorders & $17(39.5)$ & $6(30)$ & $11(47.8)$ & 0.233 \\
\hline Focal neurological deficits ${ }^{a}$ & $15(34.9)$ & $2(10)$ & $13(56.5)$ & 0.001 \\
\hline Coma & $7(16.3)$ & $3(15)$ & $4(17.4)$ & 0.832 \\
\hline Abnormal involuntary movements & $4(9.3)$ & $1(5)$ & $3(13)$ & 0.365 \\
\hline Delirium & $3(7)$ & 0 & $3(13)$ & 0.054 \\
\hline Seizure & $3(7)$ & 0 & $3(13)$ & 0.054 \\
\hline Extraneurological signs, n (\%) & $13(30.2)$ & $2(10)$ & $11(47.8)$ & 0.007 \\
\hline Uveitis & $5(11.6)$ & $1(5)$ & $5(21.7)$ & 0.114 \\
\hline Arthralgia & $7(17.1)$ & $1(5)$ & $6(26.1)$ & 0.061 \\
\hline Aphthous ulcers & $3(7)$ & 0 & $3(13)$ & 0.094 \\
\hline Skin lesions ${ }^{\mathrm{b}}$ & $4(9.3)$ & 0 & $4(17.4)$ & 0.050 \\
\hline High CRP level, ${ }^{\mathrm{C}} \mathrm{n}(\%)$ & $25(58.1)$ & $14(70)$ & $11(47.8)$ & 0.141 \\
\hline \multicolumn{5}{|l|}{ CSF analysis } \\
\hline Protein level, median (range) & $0.62(0.34-2.7)$ & $0.56(0.34-1.94)$ & $1(0.48-2.7)$ & 0.535 \\
\hline High protein level, ${ }^{\mathrm{d}} \mathrm{n}(\%)$ & $34(79)$ & $14(70)$ & $20(87)$ & 0.173 \\
\hline Low glucose level, ${ }^{\mathrm{e}} \mathrm{n}(\%)$ & $8(18.6)$ & $2(10)$ & $6(26.1)$ & 0.176 \\
\hline Cells/mm³ ${ }^{3}$ median (range) & $43(6-2,000)$ & $26(6-1,938)$ & $62(6-2,000)$ & 0.726 \\
\hline Lymphocytic meningitis, n (\%) & $32(74.4)$ & $14(70)$ & $18(78.3)$ & 0.535 \\
\hline Neutrophilic meningitis, n (\%) & $17(39.5)$ & $7(35)$ & $10(43.5)$ & 0.570 \\
\hline
\end{tabular}


Table 2. Imaging findings, treatment and follow-up

\begin{tabular}{|c|c|c|c|c|}
\hline & All $(n=43)$ & Idiopathic $(n=20)$ & SID $(n=23)$ & p-value \\
\hline Abnormal MRN findings, ${ }^{a} \mathrm{n}(\%)$ & $30 / 41(73.2)$ & $10 / 18(55.5)$ & $20 / 23(86.9)$ & 0.024 \\
\hline T2-weighted high signal lesions & $14(34.1)$ & $5(27.8)$ & $9(39.1)$ & 0.324 \\
\hline Pseudotumoural lesions & $3(7.3)$ & 0 & $3(13)$ & 0.094 \\
\hline Arachnoiditis & $7(17.1)$ & $3(16.7)$ & $4(17.4)$ & 0.832 \\
\hline Dilatation of ventricles & $2(4.9)$ & $2(11.1)$ & 0 & 0.120 \\
\hline Myelitis & $3(7)$ & 0 & $3(13)$ & 0.094 \\
\hline Vasculitis $^{b}$ & $2(4.9)$ & 0 & $2(8.7)$ & 0.177 \\
\hline ICU, n (\%) & $13(30.2)$ & $6(30)$ & $7(30.4)$ & 0.975 \\
\hline Treatment, n (\%) & $37(86)$ & $15(75)$ & $22(95.7)$ & 0.051 \\
\hline Steroids & $29(67.4)$ & $7(35)$ & $22(95.7)$ & $<0.001$ \\
\hline Antibiotics & $22(51.1)$ & $11(55)$ & $11(47.8)$ & 0.451 \\
\hline Antituberculous therapy & $6(13.9)$ & $2(10)$ & $4(17.4)$ & 0.485 \\
\hline Acyclovir IV infusion & $15(34.9)$ & $10(50)$ & $5(21.7)$ & 0.052 \\
\hline Follow up, median (range) & $32.5(1-73)$ & $19(1-60)$ & $38(14-73)$ & 0.250 \\
\hline Chronic ${ }^{c} /$ recurrent meningitis, n ( \% ) & $15(34.9)$ & $6(30)$ & $9(39.1)$ & 0.531 \\
\hline Death, n (\%) & $1(2.3)$ & $1(5)$ & 0 & 0.278 \\
\hline
\end{tabular}

$(n=3)$, undifferentiated connective tissue disease ( $n=2)$, Sjögren's syndrome $(n=1)$ and unclassified autoinflammatory syndrome $(n=1)$, which was then considered a flare-up of the underlying disease. Overall, sarcoidosis and Behçet's disease accounted for almost half of the SIDs, either revealed or complicated by aseptic meningitis.

Approximately $50 \%$ of the patients with aseptic meningitis received empirical antibiotics and/or antiviral treatment at diagnosis. All but one patient with SID received steroids as a specific therapy. Of note, steroids were also given to seven patients with idiopathic meningitis, including four with chronic and/or recurrent meningitis (Table 2 ).

Rates of intensive care unit admission, death or relapse did not differ between patients with or without SID during a median follow-up of 32.5 (1-73) months. In patients with idiopathic meningitis, no SID was diagnosed during a median follow-up of 19 (1-60) months following the diagnosis of aseptic meningitis. Only one patient died, from asbestosisrelated respiratory failure 34 months after the diagnosis of meningitis.

Key value of neurological and extraneurological signs for the diagnosis of systemic inflammatory disorders

Compared with patients with idiopathic meningitis, patients with SIDs displayed a higher frequency of neurological signs, including focal neurological deficits $(p=0.001)$, delirium $(p=0.054)$ or seizure $(p=0.054)$ at diagnosis. Of note, delirium and seizure were never observed in patients with idiopathic meningitis. Moreover, the frequency of extraneurological signs, including uveitis, arthralgia, aphthous ulcers and skin lesions, was significantly higher in patients with SIDs $(p=0.007)$. High protein $(p=0.173)$ and low glucose $(p=0.176)$ levels in CSF tended to be more frequently associated with SIDs (Table 1). Abnormal cerebral MRI findings $(p=0.024)$ were also observed more frequently in patients with SIDs (Table 2).

In the multivariate analysis, the presence of neurological and extraneurological signs were both associated with a higher probability (odds ratio [OR] [95\% CI] 6.36 [1.33-47.08] and OR [95\% CI] 13.31 [2.36-136.12], respectively) of having an SID. Overall, according to the logistic regression model, the probability of an SID in patients admitted with acute meningitis with neurological and extraneurological signs was $93.7 \%$. This fell to $14.9 \%$ in patients with acute meningitis with neither neurological nor extraneurological signs (Fig 2).

\section{Discussion}

This retrospective monocentric study was conducted to identify simple clinical clues for the diagnosis of SIDs in patients admitted for acute aseptic meningitis. From our data, a clinical triage of patients admitted for acute aseptic meningitis based on neurological and extraneurological signs helped to identify patients in whom an SID, mostly sarcoidosis or Behçet's syndrome, was probable or unlikely.

In our study, approximately $50 \%$ of patients with aseptic meningitis had an SID that was revealed by the meningitis in most cases. This frequency of SID is higher than previously reported. ${ }^{11}$ Of note, a diagnosis of aseptic meningitis is made once infection has been ruled out, and so-called 'aseptic meningitis' might in fact be caused by enteroviruses or HSV-2 in more than two-thirds of 


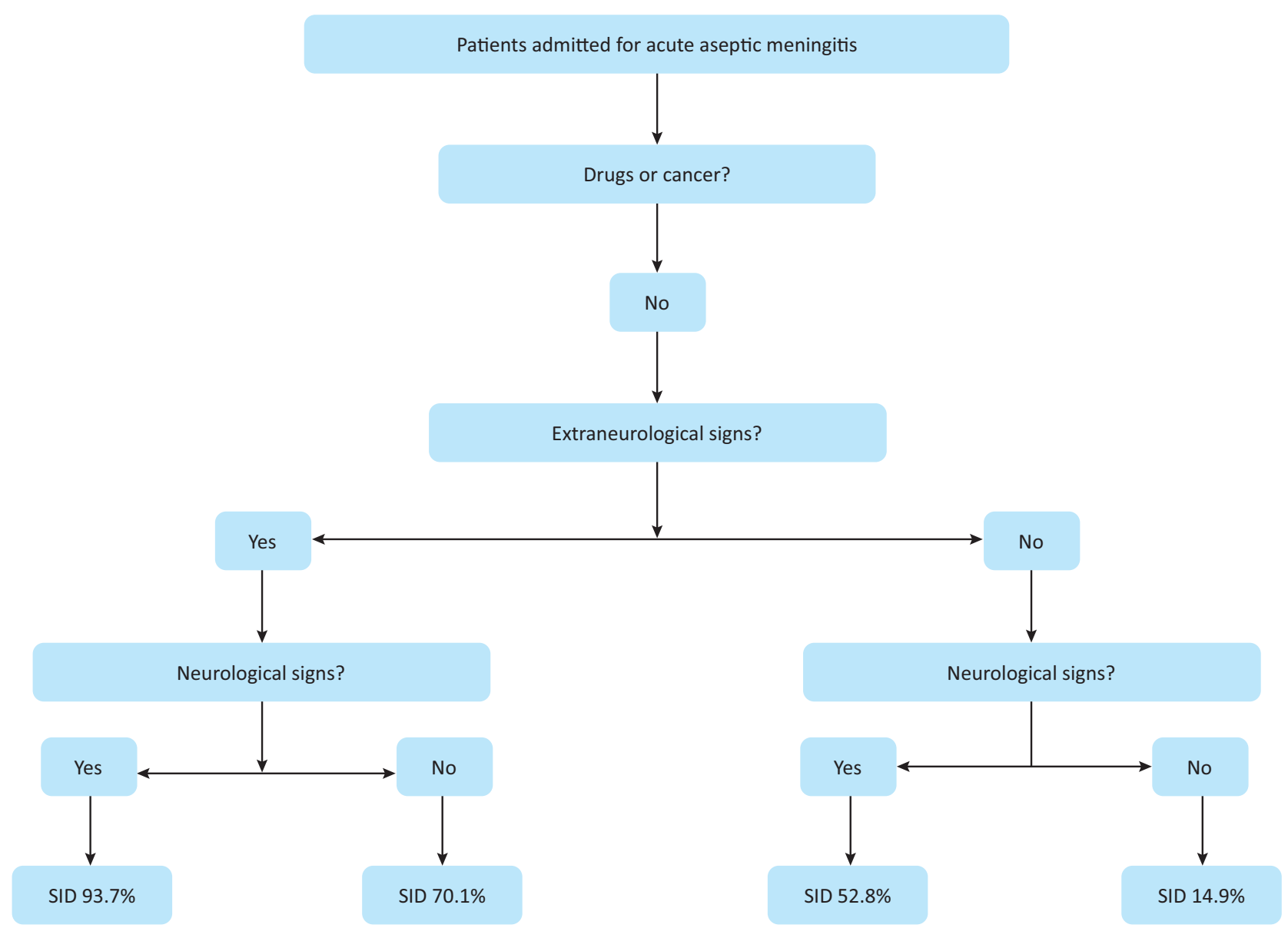

Fig 2. Probability of having a systemic inflammatory disorder (SID) according to neurological and extraneurological signs in patients admitted for acute meningitis. Neurological signs: focal neurological deficits, delirium or seizure at diagnosis. Extraneurological signs: uveitis, arthralgia, aphthous ulcers and skin lesions (ie folliculitis, malar rash, photosensitivity and erythema nodosum).

cases. ${ }^{11,13}$ Hence, the high frequency of SID observed in our study is best explained by the fact that such patients with infectious meningitis were indeed excluded, increasing the proportion with SIDs.

A key finding of our study was the high level of suspicion for SID conferred by extraneurological signs. Of note, extraneurological signs were systematically assessed in all patients admitted with acute aseptic meningitis, thus limiting bias related to the extraction of data retrospectively from medical records.

In SIDs such as sarcoidosis or Behçet's disease with CNS involvement, brain MRI is rarely normal. ${ }^{6}$ Consistent with the high frequency of neurological signs at diagnosis, abnormal MRI findings were frequently observed in patients with SIDs. Interestingly, some imaging patterns, such as pseudotumoural lesions and myelitis, were never observed in patients with idiopathic aseptic meningitis. Similarly, other groups have reported that small cortical or subcortical lesions hyperintense in T2 weighting are consistent with small vessel vasculitis and that gadolinium enhancement in T1 weighting in subarachnoid spaces suggests systemic disease rather than idiopathic meningitis. ${ }^{14,15}$ Despite a tendency for a higher protein level in CSF to be associated with SID, we did not find any significant differences in the CSF analysis between idiopathic and SIDrelated meningitis.

Some patients with idiopathic meningitis and no clear-cut SID appear to have 'borderline' characteristics, including the presence of extraneurological signs with abnormal MRI findings, with some patients requiring steroid therapy. Such findings have already been reported and these patients with steroid-sensitive meningitis might have 'undefined SID'. ${ }^{16,17}$ In our study, no SIDs were diagnosed in patients with idiopathic meningitis over a median follow-up of 19 months.

Our study has limitations that prevent the generalisability of these results. Although we enrolled all consecutive symptomatic patients with acute aseptic meningitis during a 4-year period, it is a single-centre retrospective study with a relatively small cohort. Data were extracted from medical records by investigators who were not blinded to the final diagnosis. Despite these limitations, our retrospective study points to simple clinical tools that can be used to detect a systemic cause for acute aseptic meningitis.

In conclusion, a structured clinical work-up focusing on both neurological and extraneurological features is effective to both stratify patients with acute aseptic meningitis and identify those with SIDs. 


\section{References}

1 Moris G, Garcia-Monco JC. The challenge of drug-induced aseptic meningitis. Arch Intern Med 1999;159:1185-94.

2 Chamberlain MC. Neoplastic meningitis. Neurologist 2006;12:17987.

3 Hietaharju A, Yli-Kerttula U, Hakkinen V, Frey H. Nervous system manifestations in Sjogren's syndrome. Acta Neurol Scand 1990;81:144-52.

4 Tervaert JW, Kallenberg C. Neurologic manifestations of systemic vasculitides. Rheum Dis Clin North Am 1993;19:913-40.

5 Aisen AM, Gabrielsen TO, McCune WJ. MR imaging of systemic lupus erythematosus involving the brain. Am J Roentgenol 1985;144:1027-31.

6 Wechsler B, Dell' Isola B, Vidailhet $M$ et al. MRI in 31 patients with Behcet's disease and neurological involvement: prospective study with clinical correlation. J Neurol Neurosurg Psychiatry 1993;56:793-8.

7 Kalra S, Silman A, Akman-Demir G et al. Diagnosis and management of Neuro-Behcet's disease: international consensus recommendations. J Neurol 2014;261:1662-76.

8 Segal BM. Neurosarcoidosis: diagnostic approaches and therapeutic strategies. Curr Opin Neurol 2013;26:307-13.

9 Sciascia S, Bertolaccini ML, Baldovino S et al. Central nervous system involvement in systemic lupus erythematosus: Overview on classification criteria. Autoimmun Rev 2013;12:426-9.

10 Baizabal-Carvallo JF, Delgadillo-Marquez G, Estanol B, Garcia-Ramos G. Clinical characteristics and outcomes of the meningitides in systemic lupus erythematosus. Eur Neurol 2009;61:143-8.
11 Jarrin I, Sellier P, Lopes A et al. Etiologies and management of aseptic meningitis in patients admitted to an internal medicine department. Medicine (Baltimore) 2016;95:e2372.

12 Centers for Disease Control and Prevention. Case definitions for infectious conditions under public health surveillance. MMWR Recomm Rep 1997:46:1-55.

13 Kupila L, Vuorinen T, Vainionpaa R et al. Etiology of aseptic meningitis and encephalitis in an adult population. Neurology 2006;66:75-80

14 Appenzeller S, Kobayashi E, Costallat LT et al. Magnetic resonance imaging in the evaluation of patients with aseptic meningoencephalitis and connective tissue disorders. Arq Neuropsiquiatr 2000;58:45-51.

15 Lee JH, Lee JY, Lee Y] et al. Noninfectious meningitis caused by systemic lupus erythematosus: a case series of 4 patients. J Comput Assist Tomogr 2016:40:424-7.

16 Charleston AJ, Anderson NE, Willoughby EW. Idiopathic steroid responsive chronic lymphocytic meningitis-clinical features and long-term outcome in 17 patients. Aust N Z J Med 1998:28:784-9.

17 Kinirons P, Baily C, De Gascun C, Hutchinson M. Lymphocytic meningitis: incidence, causes and outcomes over five years. Ir Med J 2005;98:238-40.

Address for correspondence: Professor Karim Sacre, Department of Internal Medicine, Bichat Hospital, APHP, 46 rue Henri Huchard, 75018 Paris, France.

Email: karim.sacre@aphp.fr 